\title{
One-Month-Old Female Baby with Symmetrical Hydrocephalus: Treatment Option
}

\author{
Muhammad Shakil Ahmad Siddiqui ${ }^{1,2}$, Khan Usmanghani ${ }^{2 *}$, Ejaz Mohiuddin ${ }^{2}$, \\ Laeequr Rahman Malik ${ }^{2}$ \\ ${ }^{1}$ Rafah-e-Aam Dawakhana Ajmali (Clinics) and Rafah-e-Aam Herbal Laboratories, Karachi, Pakistan \\ ${ }^{2}$ Faculty of Eastern Medicine, Hamdard University, Karachi, Pakistan \\ Email: *ugk_2005@yahoo.com
}

Received July 15, 2012; revised August 16, 2012; accepted August 22, 2012

\begin{abstract}
A female infant, named Anum $1.8 \mathrm{~kg}$ was born on 9th August 2007 at Jinnah Postgraduate Medical Centre (JPMC) with a big head, Occipitofrontal Circumference (OFC) $36 \mathrm{~cm}$. She was admitted on 29th August 2007 and was discharged on 5th September 2007 from Jinnah Postgraduate Medical Centre after the management of moderate birth asphyxia. Cranial ultrasound revealed moderate Hydrocephalus (symmetrical) with prominent third ventricle. Treatment and its effectiveness have been discussed.
\end{abstract}

Keywords: Hydrocephalus; Meningocele; Herbal Treatment

\section{Introduction}

Hydrocephalus is a condition where there is an excessive accumulation of cerebrospinal fluid (CSF) under pressure and at times under no pressure resulting from impaired formation and absorption of CSF. Hydrocephalus may be of two (2) types; it may be communicating (obstructive) where there is an obstruction of the ventricular system of the brain or it may be non-communicating obstructive where there is an obstruction to the flow of CSF within ventricular system. Dorsal meningocele was operated on 3rd September 2007 at Department of Neurosurgery, Jinnah Postgraduate Medical Centre (JPMC), Karachi.

\section{Case Presentation}

A term female infant of one month old (Figure 1) was born with a big head that was not noticed earlier in-utero (Figure 2). There she had convulsions and mild fever after the delivery of the baby. No abnormalities were noted except for the mucous vaginal discharge which is not unusual for a female baby.

The baby was delivered through cesarean section history at Zainab Panjwani Memorial Hospital, Nishtar Road, Karachi. On delivery the baby neither cried immediately nor did she suck. She was just found to be a small baby with a big head and dorsal region of her spine showed a sac like projection identified as meningocele. After birth the APGAR score was 2 and 4 at first and

${ }^{*}$ Corresponding author. fifth minutes respectively. The case under study belongs to congenital hydrocephalus.

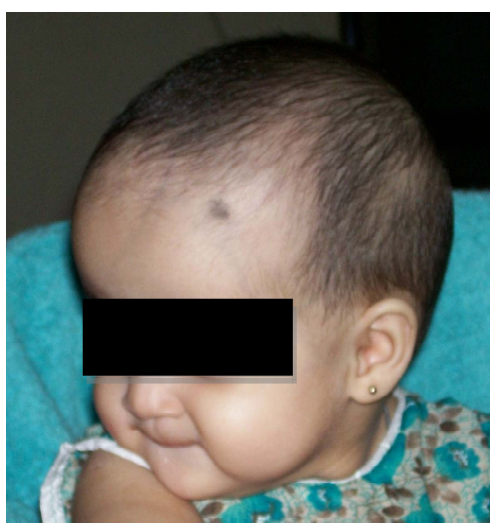

Figure 1. Hydrocephalus baby.

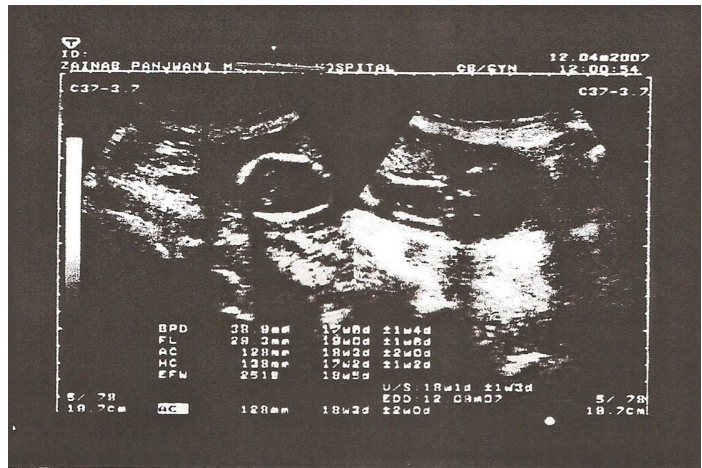

Figure 2. Ultrasound of Fetus at 18th week. 
On examination, anterior and posterior fontanelle were widen and full looking like water melon, dark brown silky hair, impaired up gaze, with abnormal head shape and large forehead, but dilatation of scalp veins and hypertonic lower extremities and the face was broadened. No other abnormal features were seen. The eyes showed setting sun sign with visible sclera above the iris. Intracranial pressure was high and nystegmus was observed.

Anthropometric measurement,

Occipitofrontal Circumference $35 \mathrm{~cm}$,

(normal 32 - 35 cm conclusion, Hydrocephalus)

Length $49 \mathrm{~cm}$,

Weight $2.19 \mathrm{~kg}$.

One systemic examination of the central nervous system, the baby was alert with partial sucking reflex and a positive glabellar reflex, rooting reflex, grasp reflex, both hand and plantar, stepping, biceps, triceps, knee, ankle, tendon and moro reflexes. The muscle tone was hypertonic.

The diagnosis was made after, cranial ultrasound scan (US) revealing huge hydrocephalus with bilateral ventriculomegally merging into one probably due to atresia of the aqueduct of sylvius. The six days later and before the discharge, the Ultra-Sound scan was repeated showing the restriction of brain cortical growth due to fluid compression.

During stay in the ward the baby was given inj vitamin $\mathrm{K}$, syp ampicillin, twice a day and syp augmentin and syp ponston once a day for four days. Moreover, the baby was kept warm with daily monitor of the respiratory rate, heart rate, and temperature and measuring of OFC. The treatment of hydrocephalus is to pass a shunt for the CSF fluid to drain into the peritoneum (ventrricoloperitoneal shunting). The parents were advised to consider adopting this procedure for which they refused, taking the responsibility themselves.

\subsection{Ultrasound of Skull}

Rt. Lat ventricle: 1.6

Rt. Hemisphere: 3.8

Left Ventricle: 1.6

Left Hemisphere: 3.7

3rd Ventricle: $1.1 \times 0.7 \mathrm{~cm}$

4th Ventricle: Not Dilated.

\subsection{Impression: Moderate Hydrocephalus (Symmetrical)}

Ultrasound of Skull:

Normal both Hemispheres

No abnormal lateral or 4th Ventricular Dilatation seen

Prominent 3rd ventricle measure $1.0 \times 0.4 \mathrm{~cm}$

No intra Ventricular Hemorrhages seen

No Midline shift seen
Normal Cerebellum and choroid plexus

Resistive Index of cerebral vessels is normal

MRI of Dorsal Spine (Full Study)

Sequences: T1 W1 sagittal and axial images were obtained.

\subsection{Findings}

There is a focal area of abnormal signal intensity which is seen in the subcutaneous region of the upper back. This is communicating with the spinal canal in the dorsal region through a defect in the posterior bony element in the upper dorsal spinal region. CSF is noted within the lesion. Minimal extension of cord tissue is also noted in this region. Findings are consistent with a dorsal meningomyelocele.

No tumor mass is seen.

Conusmedullaris terminates at D12-L1.

\subsection{Impression}

There is a focal area of abnormal signal intensity which is seen in the subcutaneous region of the upper back. This is communicating with the spinal canal in the dorsal region through a defect in the posterior bony element in the upper dorsal spinal region. CSF is noted within the lesion. Minimal extension of cord tissue is also noted in this region. Findings are consistent with a dorsal meningomyelocele.

\subsection{Hydrocephalus Herbal Formulation}

The patient was brought by the family members to Hakim Muhammad Shakil Ahmad Siddiqui at Rafah-eAam Dawakhana Ajmali (Clinics) and Rafah-e-Aam Herbal Laboratories for consultation. After reviewing the case thoroughly the patient was prescribed the herbal medicine as follows. Formulation I: Afteemoon (Cuscuta reflexa herb) $20 \mathrm{mg}$, Berg Gaozaban (Borage officinalis leaf) $20 \mathrm{mg}$, Bisfaij (Polypodium vulgare Linn root) 20 mg, Kabab Chini (Piper cubeba fruit) $20 \mathrm{mg}$, Ushba (Sarsaparilla indica herb) $20 \mathrm{mg}$, Chob Chini (Smilax chinensis root) $30 \mathrm{mg}$, Gluemundin (Sphaeranthus indicus) Aqueous ext. $20 \mathrm{mg}$.

Formulation II: Gule Surkh (Rosa demascena flower) $30 \mathrm{mg}$, Sandal safed (Santalum album wood) $30 \mathrm{mg}$, Sandal surkh (Pterocarpus santalinus wood) $30 \mathrm{mg}$, Senna (Cassia senna leaf) 40 mg, Post Balela (Terminalia belerica fruit coat) $10 \mathrm{mg}$, Sumbultib (Nardostachys jatamansi root) $10 \mathrm{mg}$, Haleela Siyah (Terminalia chebula unripe fruit) $6 \mathrm{mg}$, Post Haleela Zard (Terminalia chebula half ripe fruit coat) $6 \mathrm{mg}$.

For associated symptoms the herbal dosage form design included Laooq Khiyar Shanber for constipation, Ghutti ( Saunf, Ajowain, Podina and Ilachi) for indigestion, Sherbet Katan and Diya Quza for cough, Shobi 
Lehsan and Nukhsa Sobi, for otitis, and Laooq Sapistan and Nukhsa Nazla for cold and rhinitis.

The treatment was started on 20 September 2007 and continued up to 11 October 2008. During the treatment the following clinical features were observed. Weight gain $7.5 \mathrm{~kg}$. Setting sun sign showed improvement, there was no vomiting or fits and the baby showed improvement of normal growth. The monitoring of clinical features exhibited mild improvement between 20 September to 11 October 2007, Moderate improvement was seen between 12 October 2007 till 10 December 2007, and complete improvement was seen by 11 October 2008.

\section{Discussion}

Peach [1] has specified that the congenital hydrocephalus produces brain atrophy hence week prognosis and mental retardation is observed with the passage of time. Therefore, it is advisable to observe carefully all other system manifestation of the body along with nervous system manifestation. In this respect the CT scan and ultra sound scan should be useful tool to monitor multi-organ damage.

We had constantly monitored the patients over a period of 5 years with prescribed dosage form design of composite mixture of herbal drugs as specified in the text. However for other sign and symptoms of associated disorder beside the prescribed medicine, other herbal drugs were also administered to wipe out the malaise. As such the stunting of the baby patients was refused by the parents; therefore the patient was carefully monitored and overall performance was improved. The patients showed no mental retardation but otherwise the child is very much active and study performance is over all is good.
So indirectly it can be inferred that the different herbal drugs prescribed showed the synergistic effects both on mental and body faculties and that hydrocephalus effects was not noticeable. The herbal formulations were selected on the bases to expel cerebrospinal fluid and to expand the ventricles to reduce the pressure. However, it is very difficult to pin point the mechanism by which these herbal drugs have brought about the positive and beneficent effect on the hydrocephaly. However in this child, there was a possibility of mental retardation and delayed mile stone due to brain cortical growth failure were nullified by the use of prescribed herbal medication. The overall effects of prescribed herbal medication could be assigned as neuro degenerative disease and neuronal dysfunction treatment. As such neuro degenerative disease and neuronal dysfunction has been treated by herbal medicine and the composition comprised of Angelica sinensis, Ligusticum chuanxiong, Polygonum sibricum, Carthamus tinctorius, Astragalus mebrananaceus, Glycyrrhiza uralensis. This is given in United State Patent Xia, Patent No: US7, 416, 747 B2, date of patent: August 26, 2008. In addition drugs such as Isosorbide which produces hyperosmotic diuresis and those as such Aacetazolamide which decrease the secretion of Cerebrospinal Fluid (CSF) may be treated as temporary management of clinical situation for the treatment of hydrocephalus [2].

\section{REFERENCES}

[1] B. A. Peach, "Malformation: Morphogenesis," Archives of Neurology, Vol. 12, No. 5, 1955, pp. 527-535. doi:10.1001/archneur.1965.00460290083009

[2] R. Raza and Q. Anjum, Journal of the Pakistan Medical Association, Vol. 55, No. 11, 2005, pp. 502-507. 